\title{
Is Motivational Style Related to Sex or Gender Identity? A Study of Young Singaporeans
}

\author{
Richard Howard \\ Institute of Mental Health, Nottingham
}

\begin{abstract}
This study investigated whether individual differences in motivational style, as conceptualized and operationalized in reversal theory, are more closely linked to biological sex (male or female) or gender identity. The overarching hypothesis was that gender identity, but not sex, would be reflected in different motivational styles. In a mixed-sex sample of Singaporean students, motivational style was measured using the Apter Motivational Style Profile - Revised (AMSP-R). The Singapore Androgyny Inventory was used to classify participants as masculine, feminine, androgynous, or undifferentiated. Confirming the hypothesis, motivational style profiles reflected gender identity, not sex. Masculine and androgynous participants scored higher than feminine participants on a majority of AMSP-R scales, while undifferentiated participants scored uniformly low. Consistent with previous findings on gender identity in Singaporeans, a majority of both males and females did not show sex-congruent gender traits. The AMSP-R profiles of the sample as a whole reflected core cultural values of Singapore: desire for progress and achievement; to fit in and seek structure through rules, customs, and routines; and to achieve harmony and intimacy with others. Results support the dissociation of sex and gender cross-culturally and are compatible with the idea that individual differences in gender identity may at root reflect individual differences in motivational state.
\end{abstract}

Keywords: sex, gender, motivational style, Singapore

At the conclusion of his recent theoretical paper Apter (2015) highlighted some outstanding questions in relation to personality. In particular, he asked: "Do individuals tend to experience some combinations of motivational states more than other combinations? Indeed, do they experience certain clusters of states ... more frequently than other combinations or arrays? If so, are there cultural forces at work, with tendencies to certain pairings occurring more frequently within one culture than within another?" (p.8). An important crosscultural variable is that of gender identity/role, which, unlike biological sex, reflects " ... the sameness, unity and persistence of one's individuality as male, female, or androgynous, in greater or lesser degree, especially as it is experienced in self-awareness and behavior." (Money, 1992, p.6). Gender identity/role is an important construct in relation to personality and to reversal theory constructs in particular, for several reasons, outlined below.

First, gender identity has been said to be the "lens" through which an individual interprets, classifies, and processes people, behaviors and attributions (Wharton, Day et

Correspondence concerning this article should be addressed to Richard Howard, Institute of Mental Health, University of Nottingham Innovation Park, Jubilee Campus, Triumph Road, Nottingham, NG7 2TU, UK. E-mail: richard.howard@ nottingham.ac.uk al., 2014). It would make intuitive sense, therefore, if motivational state were related to gender identity rather than to biological sex. In the final analysis, gender identity may be subsumable under the rubric of motivational state as conceptualized in reversal theory. Differently stated, a person's gender identity (experiencing oneself as masculine, feminine, or androgynous) may ultimately reflect the way in which the motives for one's actions are experienced.

Second, emotions, a central concern of reversal theory (Apter, 1989), are more closely related to gender identity than to biological sex. For example, the experience and expression of anger is unrelated to biological sex but clearly associated with a masculine gender identity (Milovchevich, Howells, Drew, \& Day, 2001; Wharton, Day et al., 2014). Antisocial personality traits have been reported to be positively associated with a masculine gender identity and negatively associated with a feminine gender identity (Hamburger, Lilienfeld, \& Hogben, 1996).

Third, marked differences are seen across different cultures in the value they give to masculine, as contrasted with feminine or androgynous, psychological traits, with predominantly "non-masculine" societies (e.g. the Semai of Malaysia) at one pole and predominantly masculine societies (e.g. the Truk and the Samburu) at the opposite pole (Gilmore, 1990). Gilmore speculates that masculine ideologies are adaptations to the social environment specific to each 
culture: " ...the harsher the environment and the scarcer the resources, the more manhood is stressed as inspiration and goal" (Gilmore, 1990, p. 224). Singapore is of particular interest in this regards, since its core cultural values are industry and social harmony (Chang, 1996). Studies (e.g. Chang, 1997; Ward, 2000) suggest that gender traits are evenly distributed across the sexes in Singaporeans, with the majority of individuals falling into the categories labelled androgynous and undifferentiated. The androgenous displays a high degree of both masculine (e.g. "dominant", "forceful") and feminine (e.g. "compassionate", "tender", "affectionate") traits, while the undifferentiated display a lack of both masculine and feminine traits. This uncoupling of sex and gender in Singapore makes it an ideal locale for investigating gender effects on motivational state variables, since gender identity as an independent variable is relatively uncorrelated with, and hence not confounded by, biological sex. If the primary determinant of motivational state variables is gender rather than sex, one would clearly predict an absence of sex differences in motivational state variables, but clear genderrelated differences.

My study addressed this possibility by examining the relationship between participants' sex (male or female), as contrasted with their gender identity/role (masculine, feminine, androgynous, or undifferentiated) and the various measures of the Apter Motivational Style Profile (AMSP-R), developed to provide a comprehensive profile of an individual's dominance and salience within each domain (Apter, Mallows, \& Williams, 1998). The overarching hypothesis was that gender identity, but not sex, would be reflected in different AMSP-R profiles and that gender identity, measured by the Singapore Androgyny Inventory (Ward, 2000), would account for a greater proportion of the variance in AMSP-R measures than sex.

Several subsidiary hypotheses were tested:

1. Since achievement motivation has been found to be related to a masculine gender identity in Singaporeans (Chang, 1997), and since achievement is a core value of the telic state, a sub-group having a predominantly masculine gender identity will score higher on telic dominance than a sub-group identifying as feminine.

2. Since risk-taking and rebelliousness are traits that have traditionally been associated with being male, the sub-group identifying as masculine will score higher on negativism dominance than the sub-group identifying as feminine.

3. Since a desire for power is conceptually related to selforiented mastery and men have traditionally been regarded as having a greater need for power than women, the masculine group would score higher than the feminine group on selforiented mastery.

4. Since the need for establishing, maintaining, or restoring meaningful social relationships is greater in women than in men (Spence \& Helmreich, 1983) and is conceptually re- lated to other-oriented sympathy, the sub-group identifying as feminine would score higher than the masculine group on other-oriented sympathy.

A secondary aim of the present study was to take a "snapshot" of the motivational style of Singaporeans, to see whether this would reflect what are regarded as their core cultural values, namely industry and social harmony (Chang, 1996).

\section{Method}

\section{Participants}

Participants were 266 (118 male and 148 female) students from the National University of Singapore (NUS). Mean age was 21.6 years $(S D=1.3)$. Chinese respondents comprised $96.0 \%$ of the sample, Malays $2.0 \%$, Indians $1.6 \%$, and others $0.4 \%$. Only Singaporean (by nationality) participants were recruited, since the Singapore Androgyny Inventory was designed for use with Singaporean samples. Participants were from various university faculties: Arts and Social Sciences (28.7\%), Business (13.9\%), Engineering (17.9\%), Science (29.5\%), Computing (8.4\%), and Medicine (1.6\%).

\section{Procedure}

Participants were recruited by email from among the NUS undergraduate population and were administered two questionnaires - the Singapore Androgyny Inventory (Ward, 2000) and the Apter Motivational Style Profile - Revised (AMSP-R: Apter International, 2004), both in electronic form. Participants returned their completed androgyny inventory via email, while AMSP-R was administered, and the data collated, by Apter International (http://www.apterdevelopment.com). Participants were given the option of using a pseudonym in order to ensure anonymity. They were sent, by email, a feedback sheet detailing their gender identity type (based on the androgyny inventory), as well as a brief description of their motivational style profile (based on the AMSP-R).

\section{Materials}

Singapore Androgyny Inventory. The Singapore Androgyny Inventory, developed by Ward (2000), is a modification of Bem's Sex Role Inventory (BSRI: Bem, 1974). It comprises three 15-item subscales: Masculine (M), Feminine $(\mathrm{F})$ and Neutral $(\mathrm{N})$. The $\mathrm{M}$ and $\mathrm{F}$ scales reflect beliefs about gender-typed attributes of males and females, respectively, in the Singapore context. Participants rated the extent to which they think each item describes them, on a sevenpoint scale ranging from "never or almost never true" to "almost always true". Although only the M and F subscales are required for the assessment of gender type, the 15 items from the $\mathrm{N}$ subscale function as fillers. The order of the items was 


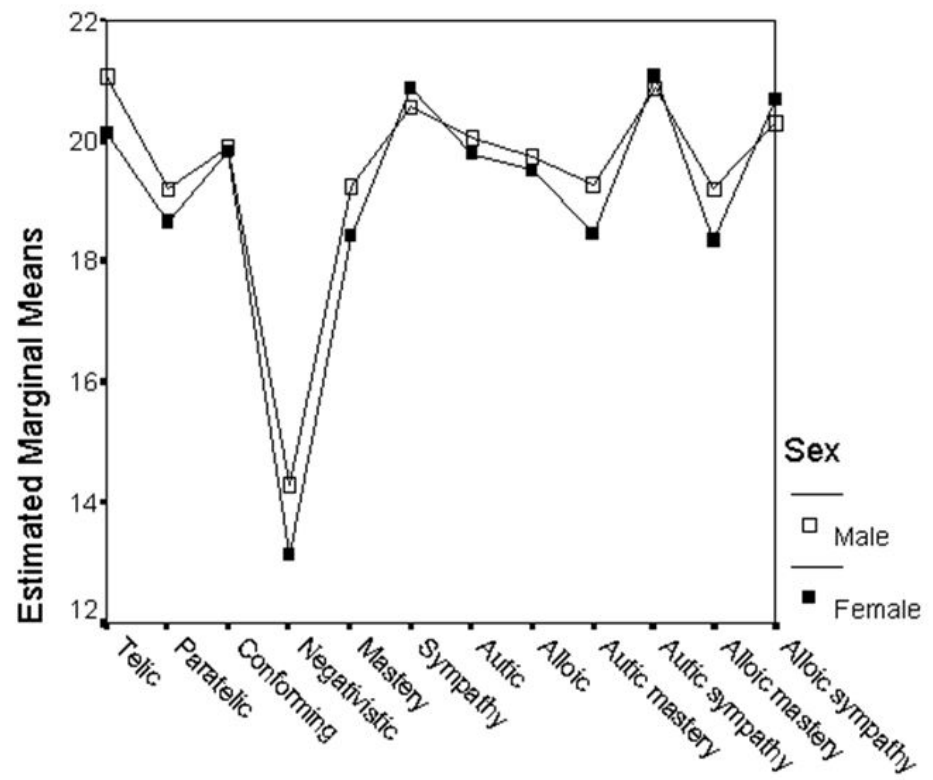

Metamotivational states

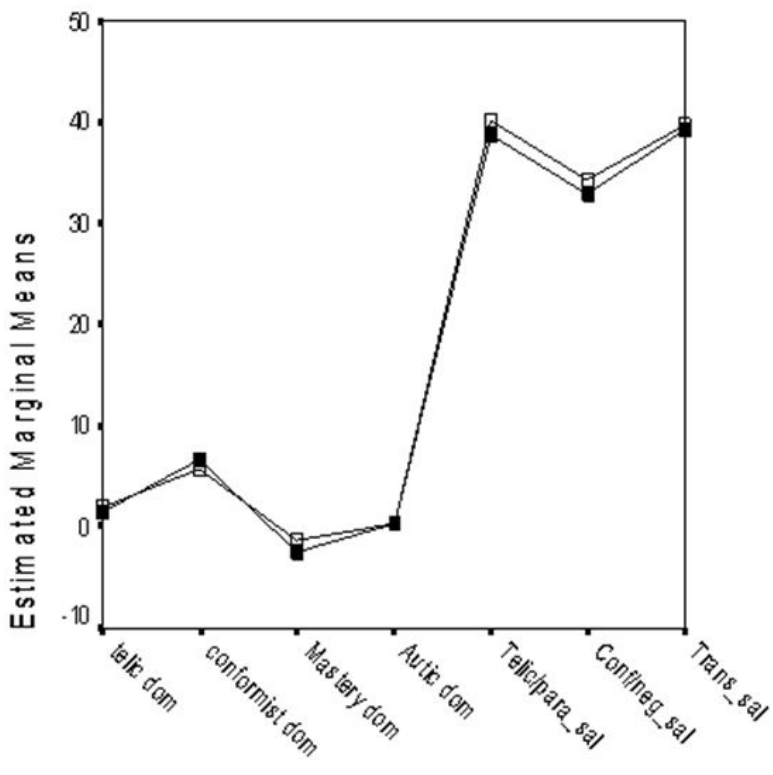

Dominance/ Salience

Figure 1. Motivational state profiles in males and females. Left figure: metamotivational states. Right figure: motivational dominance/salience.

randomly arranged to prevent participants from identifying the category of the items.

Each respondent receives two scores: one for feminine gender identity (based on the total score for the feminine subscale) and one for masculine gender identity (based on the total score for the masculine subscale). The masculine and feminine scales were scored unidirectionally by calculating the mean masculinity and femininity scores over the 15 corresponding items. Combinations of the two scores produce four different gender types: masculine (high $\mathrm{M}$, low F), feminine (low M, high F), androgynous (high M, high F) and undifferentiated (low M, low F). Assignment of participants to gender type was carried out using the median split technique, which dichotomized the variables (low and high masculinity, low and high femininity). The median for the masculinity score was 61 , while the median for the femininity score was 64 . Before assigning participants to the four gender groups, those cases $(N=15)$ with missing values on the androgyny inventory were deleted, reducing the sample size to 251 . The deleted cases appeared to be randomly distributed among the four groups. Cronbach alpha scores were acceptable and similar to those obtained by Ward (2000): .91 for the M subscale, .83 for the F subscale, and .83 for the $\mathrm{N}$ subscale.

Apter Motivational Style Profile. The revised Apter Motivational Style Profile (AMSP-R) is a shortened version of the Motivational Style Profile (Apter \& Desselles, 2001). It is designed to provide a comprehensive profile of the respondent's motivational style. It yields measures of the eight motivational states, the corresponding dominance and salience measures, as well as the four combinations derived from the mastery-sympathy and self-oriented/other-oriented pairs (see Appendix for summaries of the measures). Respondents answer 40 items in terms of how often they experience certain things on a six-point rating scale (never, seldom, sometimes, often, very often, always). The items in the AMSP-R are simple, easy to comprehend, and relatively abstract, facilitating its use in different cultures. The AMSP-R scales are reported to have fair to good internal homogeneity (Cronbach's alphas ranging from .59 to .86 for a UK and US general sample) and test-retest reliability (test-retest correlations ranging from .61 to .86) (Apter International, 2004).

The dominance measures are obtained by subtracting the score on one member of a pair from that of the other member. For example, telic dominance is obtained by subtracting the paratelic score from the telic score. A positive telic dominance score indicates that the individual is telic dominant; a negative score, that the individual is paratelic dominant. Adding the scores for the paired subscales (e.g. telic and paratelic) and dividing the sum by two to obtain an average, yields a salience score for the pair. Due to the conceptual overlap between the mastery-sympathy and selforiented/other-oriented pairs, a single score of transactional salience is computed to represent the two pairs. Thus, transactional salience deals with the different ways of experiencing transactions with others. Since each of the subscales is 
Table 1

Means of motivational style subscale scores.

\begin{tabular}{lrrccccc}
\hline Subscales & $M_{\text {Pooled }}$ & $S E$ & $99.94 \%$ CI & $M_{\text {Masc }}$ & $M_{\text {Fem }}$ & $M_{\text {Andro }}$ & $M_{\text {Undiff }}$ \\
\hline Telic & 20.58 & 0.23 & {$[19.80,21.36]$} & $22.36^{*}$ & $19.28^{*}$ & $22.13^{*}$ & $18.55^{*}$ \\
Paratelic & 18.90 & 0.17 & {$[18.32,19.49]$} & $20.25^{*}$ & $18.25^{*}$ & $19.97^{*}$ & $17.16^{*}$ \\
Conformist & 19.86 & 0.15 & {$[19.35,20.38]$} & $19.11^{*}$ & $21.08^{*}$ & 20.23 & $19.03^{*}$ \\
Negativistic & 13.65 & 0.18 & {$[13.01,14.29]$} & $14.93^{*}$ & $12.23^{*}$ & $14.35^{*}$ & 13.08 \\
Mastery & 18.80 & 0.15 & {$[18.29,19.31]$} & $19.96^{*}$ & $17.95^{*}$ & $20.35^{*}$ & $16.94^{*}$ \\
Sympathy & 20.77 & 0.17 & {$[20.17,21.37]$} & 20.65 & 21.37 & $22.14^{*}$ & $18.92^{*}$ \\
Self-oriented & 19.93 & 0.18 & {$[19.33,20.54]$} & $21.01^{*}$ & 19.56 & $21.21^{*}$ & $17.96^{*}$ \\
Other-oriented & 19.63 & 0.17 & {$[19.06,20.21]$} & 19.60 & 19.76 & $21.28^{*}$ & $17.90^{*}$ \\
Self-oriented mastery & 18.86 & 0.18 & {$[18.23,19.49]$} & $20.77^{*}$ & $17.70^{*}$ & $20.42^{*}$ & $16.56^{*}$ \\
Self-oriented sympathy & 21.01 & 0.25 & {$[20.15,21.86]$} & 21.25 & 21.42 & $22.00^{*}$ & $19.36^{*}$ \\
Other-oriented mastery & 18.74 & 0.20 & {$[18.06,19.41]$} & 19.15 & 18.20 & $20.28^{*}$ & $17.31^{*}$ \\
Other-oriented sympathy & 20.53 & 0.18 & {$[19.91,21.16]$} & 20.05 & $21.31^{*}$ & $22.28^{*}$ & $18.48^{*}$ \\
Telic dominance & 1.68 & 0.28 & {$[0.70,2.65]$} & 2.11 & 1.03 & 2.17 & 1.39 \\
Conformist dominance & 6.22 & 0.27 & {$[5.29,7.14]$} & $4.18^{*}$ & $8.84^{*}$ & 5.88 & 5.95 \\
Mastery dominance & -1.97 & 0.17 & {$[-2.56,-1.38]$} & $-0.69^{*}$ & $-3.41^{*}$ & -1.79 & -1.98 \\
Self-oriented dominance & 0.30 & 0.20 & {$[-0.40,1.01]$} & $1.41^{*}$ & -0.20 & -0.08 & 0.06 \\
Telic/paratelic salience & 39.49 & 0.29 & {$[38.50,40.48]$} & $42.61^{*}$ & $37.53^{*}$ & $42.10^{*}$ & $35.70^{*}$ \\
Conformist/negativistic salience & 33.51 & 0.20 & {$[32.81,34.22]$} & 34.05 & 33.31 & $34.58^{*}$ & $32.11^{*}$ \\
Transactional salience & 39.57 & 0.27 & {$[38.62,40.51]$} & $40.61^{*}$ & 39.32 & $42.49^{*}$ & $35.86^{*}$ \\
\hline
\end{tabular}

$*$ = means that fall outside the confidence intervals. $\mathrm{M}_{\mathrm{Masc}}=$ mean score for the masculine group. $\mathrm{M}_{\mathrm{Fem}}=$ mean score for the feminine group. $\mathbf{M}_{\text {Andro }}=$ mean score for the androgynous group. $\mathbf{M}_{\text {Undiff }}=$ mean score for the undifferentiated group.

independent of all the other subscales, the AMSP-R is a nonipsative instrument.

Data screening and analysis. Various SPSS programs were used for data screening. Two cases from the masculine group with univariate outliers were deleted, thus reducing the number of participants in the masculine group to 61 . No other univariate or multivariate outliers were detected among the remaining 249 participants, with $p=.001$. After deletion of cases with missing data, assumptions regarding normality of sampling distributions, homogeneity of variance-covariance matrices, linearity, and multicollinearity were met.

SPSS GLM was used to carry out profile analysis of AMSP-R data, first using sex as a grouping variable and then using gender identity as a grouping variable. Profile analysis was applied to AMSP-R data to determine whether groups (e.g. males vs. females) differed in their AMSP-R profiles, including the parallelism of their profiles, the overall difference between groups (levels), the similarity across dependent variables (flatness), and the statistical significance of any differences found. When using profile analysis, the main description of results is a graph of the profiles for each group, with the means for each dependent variable plotted, allowing for comparison of the plots for each group (Tabachnick \& Fidell, 2001).

\section{Results}

\section{Gender Identity by Sex}

The pooled sample androgyny inventory means for the masculine, feminine, and gender-neutral subscales were 4.02 $(S D=.86), 4.24(S D=.74)$, and $4.65(S D=.64)$, respectively. Consistent with Bem's contention that masculinity and femininity are orthogonal clusters of traits, the two subscales showed a non-significant correlation $(r=.08)$. Ttests revealed significant sex differences in mean masculinity and femininity scores for males and females. Males ( $M=4.26)$ scored higher in masculinity than did females, $M=3.84 ; t(247)=3.88, p<.001$, and females $(M=4.34)$ scored higher in femininity than males, $M=4.11 ; t(247)=$ $2.45, p<.05$.

\section{AMSP-R Profiles: Sex as a Grouping Variable}

The profiles of male and female participants are shown in Figure 1, where it may be seen that the profiles are virtually identical. Using Wilks's criterion, the profiles of males and females participants demonstrated significant parallelism, $F(8,240)=3.04, p=.003$, partial $\eta^{2}=.092$. For the levels test, no reliable differences were found between the sexes when scores were averaged over all the subscales, $F(1,247)=3.23, p=.074$. When averaged across the two groups, the subscales were found by Hotelling's criterion to 


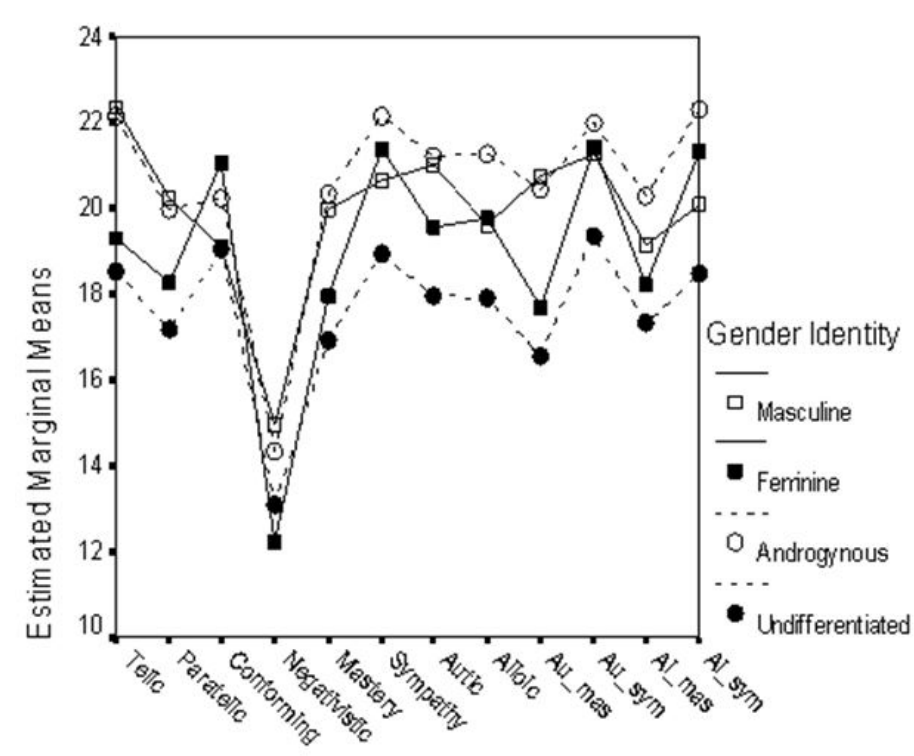

Metamotivational states

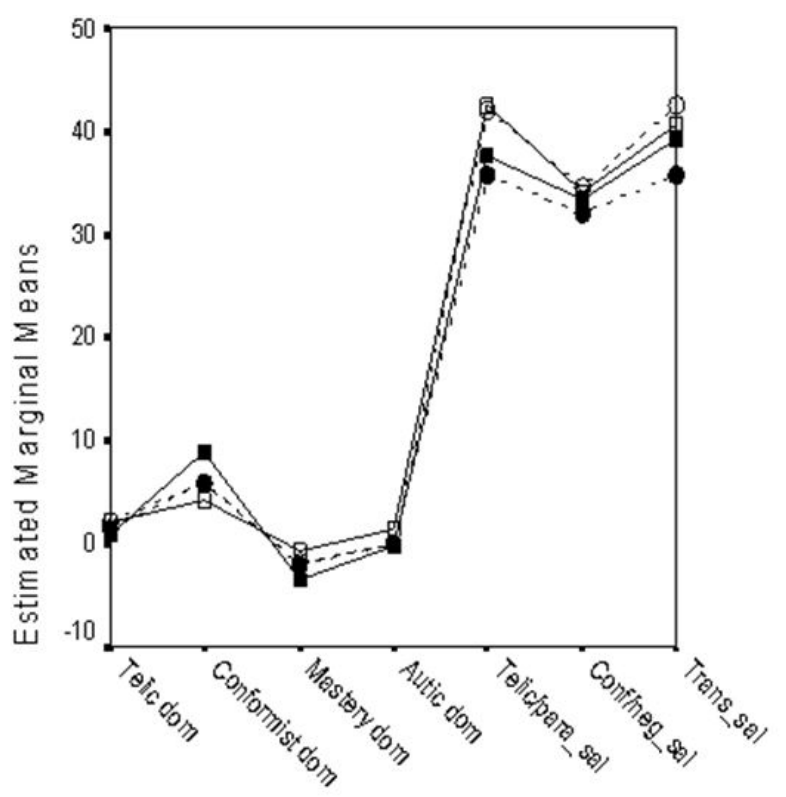

Dominance/ Salience

Figure 2. Motivational state profiles in the four gender identity groups. Left of figure: metamotivational states. Right of figure: motivational dominance/salience.

deviate significantly from flatness, $F(8,240)=4135.25, p<$ .001 , partial $\eta^{2}=.99$.

To evaluate deviation from parallelism of the profiles, confidence limits were calculated around the mean of the profile for the two groups combined (pooled mean, MPooled). Alpha error for each confidence interval was set at .0013 to achieve an experimentwise error rate of $5 \%$. Therefore, $99.87 \%$ limits were evaluated for the pooled profile. However, neither group had means that fell outside these limits.

\section{AMSP-R Profiles: Gender Identity as a Grouping Vari- able}

Profile analysis was also conducted on the second grouping variable, gender identity (masculine, feminine, androgynous, undifferentiated). The profiles for the 4 groups are shown in Figure 2. Using Wilks's criterion, the profiles of these four gender identity groups deviated significantly from parallelism, $F(24,691)=8.42, p<.001$, partial $\eta^{2}=.22$. For the levels test, reliable differences were found among the four groups when scores were averaged across all 20 subscales, $F(3,245)=33.19, p<.001$, partial $\eta^{2}=.29$. When averaged over groups, subscales were found by Hotelling's criterion to deviate significantly from flatness, $F(8,238)=$ $5169.8, p<.001$, partial $\eta^{2}=.99$. The proportion of variance accounted for by gender identity (represented by partial $\eta^{2}$ ), with regards to the differences in the measures of the AMSP-R, was greater than the proportion of variance accounted for by sex.
To evaluate deviation from parallelism of the profiles, confidence limits were calculated around the mean of the profile for the four groups combined (pooled mean, MPooled). Alpha error for each confidence interval was set at .00065 to achieve an experiment-wise error rate of $5 \%$. Therefore, 99.94\% limits were evaluated for the pooled profile. As shown in Table 1, for all of the subscales (with the exception of telic dominance), one or more groups had means that fell outside these limits.

The levels hypothesis addresses the same question as the between-subjects main effect in a repeated-measures ANOVA. From the data provided in Table 2, it may be observed that undifferentiated individuals consistently had the lowest means on all subscales, except for the negativistic subscale and the four dominance scores. Androgynous individuals had reliably higher means on all subscales (with the exception of the conformist subscale and the four dominances) than that of the pooled groups (MPooled).

A series of post-hoc planned comparisons was conducted using the Tukey HSD test to contrast AMSP-R scores obtained by the four gender identity groups. The significance level was set at .00044 to achieve an experimentwise error of $5 \%$ (as well as to reduce the probability of Type I error). The resultant mean difference scores comparing each group with every other group are shown in Table 2 . It may be seen that the masculine group scored significantly higher than the feminine group on the following subscales: telic, paratelic, negativistic, mastery, self-oriented mastery, mastery dominance, 
Table 2

Mean differences in motivational style scores between gender identity groups.

\begin{tabular}{|c|c|c|c|c|c|c|}
\hline \multirow[b]{2}{*}{ Subscales } & \multicolumn{5}{|c|}{ Mean difference $(x-y)$} & \multirow[b]{2}{*}{$M_{A}-M_{U}$} \\
\hline & $M_{M}-M_{F}$ & $M_{M}-M_{A}$ & $M_{M}-M_{U}$ & $M_{F}-M_{A}$ & $M_{F}-M_{U}$ & \\
\hline Telic & $3.08 * * *$ & 0.23 & $3.81 * * *$ & -2.85 & 0.73 & $3.59 * * *$ \\
\hline Paratelic & $2.00 * * *$ & 0.28 & $3.09 * * *$ & $-1.72 * *$ & 1.09 & $2.81 * * *$ \\
\hline Conformist & $-1.96 * * *$ & -1.12 & 0.08 & 0.84 & $2.05 * * *$ & 1.20 \\
\hline Negativistic & $2.70 * * *$ & 0.58 & $1.86^{* *}$ & $-2.12 * * *$ & -0.84 & 1.27 \\
\hline Mastery & $2.01 * * *$ & -0.39 & $3.02 * * *$ & $-2.40 * * *$ & 1.02 & $3.41 * * *$ \\
\hline Sympathy & -0.72 & -1.49 & $1.73 * *$ & -0.77 & $2.45 * * *$ & $3.22 * * *$ \\
\hline Self-oriented & 1.45 & -0.20 & $3.05 * * *$ & $-1.65 * *$ & $1.60 * *$ & $3.25 * * *$ \\
\hline Other-oriented & -0.16 & $-1.68 * *$ & $1.70 * *$ & $-1.53 * *$ & $1.86 * * *$ & $3.38 * * *$ \\
\hline Self-oriented mastery & $3.07 * * *$ & 0.35 & $4.21 * * *$ & $-2.71 * * *$ & 1.14 & $3.85 * * *$ \\
\hline Self-oriented sympathy & -0.18 & -0.75 & 1.89 & -0.58 & 2.06 & $2.64 * *$ \\
\hline Other-oriented mastery & 0.94 & -1.14 & $1.84 * *$ & $-2.08 * *$ & 0.89 & $2.97 * * *$ \\
\hline Self-oriented sympathy & -1.26 & $-2.23 * * *$ & 1.56 & -0.97 & 2.83 & $3.80 * * *$ \\
\hline Telic dominance & 1.08 & -0.05 & 0.72 & -1.14 & -0.36 & 0.78 \\
\hline Conformist dominance & $-4.66 * * *$ & -1.70 & -1.77 & $2.96 * * *$ & $2.89 * * *$ & -0.07 \\
\hline Mastery dominance & $2.73 * * *$ & 1.10 & 1.30 & $-1.62 * *$ & -1.43 & 0.19 \\
\hline Self-oriented dominance & 1.61 & 1.48 & 1.35 & -0.12 & -0.26 & -0.14 \\
\hline Telic/paratelic salience & $5.08 * * *$ & 0.51 & $6.90 * * *$ & $-4.57 * * *$ & 1.83 & $6.40 * * *$ \\
\hline Conformist/negativistic salience & 0.74 & -0.53 & $1.94 * *$ & -1.27 & 1.2 & $2.47 * * *$ \\
\hline Transactional salience & 1.29 & -1.89 & $4.75^{* * *}$ & $-3.17 * * *$ & $3.46 * * *$ & $6.63 * * *$ \\
\hline
\end{tabular}

$* *=p<.01 ; * * *=p<.001$

$\mathrm{M}_{\mathrm{M}}=$ mean score for the masculine group. $\mathrm{M}_{\mathrm{F}}=$ mean score for the feminine group. $\mathrm{M}_{\mathrm{A}}=$ mean score for the androgynous group. $\mathrm{M}_{\mathrm{U}}=$ mean score for the undifferentiated group.

and telic/paratelic salience; it scored significantly lower than the feminine group on the conformist and conformist dominance scales. Both the masculine and androgynous groups scored significantly higher than the undifferentiated group on a majority of subscales. The scores obtained by the masculine and androgynous groups did not differ significantly, with one exception: the androgynous group were significantly more other-oriented and scored significantly higher on other-oriented sympathy. In contrast, the androgynous group scored significantly higher than the feminine group (and in the masculine direction) on the negativistic, mastery, selforiented mastery, telic/paratelic salience, and transactional salience subscales. The feminine group had significantly higher means than the undifferentiated group on the conformist, sympathy, other-oriented, and transactional salience subscales.

Finally, it may additionally be seen from the pooled means shown in Table 1 that the sample as a whole (irrespective of gender type) was telic dominant, conformist dominant, and sympathy dominant (as implied from the negative scores on mastery dominance).

\section{Discussion}

The main hypothesis of this study, that gender identity, but not sex, would significantly contribute to differences in motivational style as measured by the AMSP-R, found over- whelming support. Gender identity clearly accounted for a far greater proportion of the variance in AMSP-R measures than did biological sex.

In addition, three subsidiary hypotheses, predicting that the masculine group would score higher on the telic, negativistic, and self-oriented mastery subscales than the feminine group, were supported. Both the masculine and androgynous groups reported higher means for the telic, negativistic, and mastery subscales than the feminine and undifferentiated groups. This was likely due to differences in masculinity, since both masculine and androgynous individuals had higher, and both feminine and undifferentiated individuals lower, levels of masculinity. However, the fourth subsidiary hypothesis, that the feminine group would score higher than the masculine group on other-oriented sympathy, was not supported. While the feminine group scored higher than the masculine group, the difference was not significant. Instead, it was the androgynous group that scored highest, and significantly higher than the masculine group.

Results of this study extend those of previous studies that have examined personality attributes such as achievementorientation (Chang, 1996), empathy (Karniol et al., 1997), learning style (Severiens \& Ten Dam, 1997) and anger (Milovchevich et al., 2001; Wharton et al., 2014), in suggesting that males and females do not differ significantly in their experience of motivation, and that motivational states 
are related to gender identity, but not sex. It appears that outside the confines of aggressiveness and casual sexuality (varieties of sexual activity occurring outside of romantic relationships that imply an absence of commitment, emotional attachment, or familiarity between sexual partners), where males score higher, and anxiety and nurturance, where females score higher (Larsen \& Buss, 2002), gender, rather than sex, is a far more important correlate of human personality. Moreover, results from the present study suggest the possibility that any apparent sex difference in personality variables found in Western samples - e.g. the above-mentioned differences suggested by Larsen \& Buss (2002) - may be related to gender rather than sex. This is because sex and gender may be more closely aligned (and therefore confounded) in Western than in non-Western cultures. Clearly, regardless of the culture in which a study is conducted, apparent sex differences cannot be attributed unambiguously to either sex or gender unless both sex and gender are considered.

Unlike sex, gender is cross-culturally variable. Gilmore (1990), for example, has, through cross-cultural studies of masculinity, documented the existence of a masculinity continuum across human societies, with predominantly "nonmasculine" societies at one pole, and predominantly masculine societies at the opposite pole. What determines these culturally specific patterns of masculinity is open to conjecture. However, as argued by Apter (2015), societies likely differ in the values they deem important and encourage in individuals, albeit they are constrained by the given set of values proposed in reversal theory.

Results of this study support previous studies by Chang (1997) and Ward (2000) in suggesting that the sexes in Singapore are relatively undifferentiated by gender compared with the West. Despite males and females showing significant differences in gender traits in the sex-congruent direction (males more masculine, females more feminine), the $a b$ solute differences were very small (e.g. a difference of only 0.23 in the mean Femininity score between males and females) and comparable to those reported in Ward's (2000) study. As in Ward (2000), around half the men and women were cross-gendered, androgynous, or undifferentiated. Females, in particular, showed a strong tendency to be either androgynous or undifferentiated, which is again consistent with previous findings. Chang (1997) found that threequarters of females were either androgynous or undifferentiated, and only $12 \%$ were feminine. It seems that in terms of Gilmore's (1990) cultural continuum of gender, Singapore is a relatively androgynous society, falling towards the nonmasculine pole of the masculinity continuum.

The overall motivational style profile found in the current Singapore sample, based on the dominance scores and disregarding gender differences, can be characterized as telic dominant, conformist dominant, and sympathy dominant. Hence, the typical young Singaporean presents as being pre- dominantly motivated by a desire for progress and achievement; by a desire to fit in and seek structure through rules, customs and routines; and by a desire to achieve harmony and intimacy with others. Interestingly, overall young Singaporeans do not present as either self-oriented or otheroriented, with the exception of the masculine gender type who are predominantly self-oriented and appear to be motivated by a desire for self-actualization. This concurs with findings by Hwang, Francesco, and Kessler (2003) that among business students from Singapore, Hong Kong and the United States, Singaporeans were the most individualistic. Whether the motivational pattern found here to characterize Singaporeans generalizes to Singaporeans in general is questionable, but it is at least consistent with Chang's (1996) comment in relation to her findings in Singaporeans: "Industry and social harmony were highly endorsed by Singaporean subjects as the more important values" (p.222). She comments further that the prevailing value structure of Chinese Singaporeans is one that emphasizes achievement (task-oriented instrumentality) and civic harmony (social/expressive values). Thus it is plausible to suggest that telic dominance and sympathy dominance were observed in all four gender identity groups because of the prevalence of these underlying traditional values in Singaporean society.

\section{Limitations of the study}

Chinese Singaporeans were relatively over-represented, and Malays and Indians under-represented, in this sample relative to their representation in the community at large, where Chinese comprise $76.8 \%$ of the population and Malays and Indians $13.9 \%$ and $7.9 \%$ respectively (Singapore Department of Statistics, 2001, p. viii). Care must be taken not to over-generalize results from the present study to the Singaporean population as a whole. It is moreover questionable whether the motivational pattern found here to characterize (predominantly Chinese) Singaporean students generalizes to Singaporeans of the same age who are not students, or to Singaporeans in general. While there is a constellation of personality traits that appears to characterize Singaporeans as a whole, personality traits also differ between Singaporean ethnicities (Cheung, Cheung, Howard, \& Lim, 2006). Finally, the data examined in the current study were wholly cross-sectional and do not permit any causal inferences to be made. The question of the precise nature of the relationship between gender identity and motivational state remains unresolved. Are they causally related, and, if so, how? In particular, which has causal primacy? Or alternatively, are genderrelated traits simply - as the current results might be taken to imply - translatable into the reversal theory language of motivational states and the values that they reflect? In other words, do gender-related traits fundamentally reflect differences in motivational state? These questions remain to be addressed in the future. 


\section{Conclusion}

This study demonstrates that motivational style as conceptualized in reversal theory is related to gender, rather than sex, in a society (Singapore) where the sexes are relatively ungendered. The question of possible differences in motivational style between the ethnic groups in Singapore - Chinese, Indians and Malays - remains to be explored in future research, using samples whose composition better represents Singaporean minority ethnic groups. The current results are compatible with the idea that individual differences in gender identity may at root reflect individual differences in motivational state.

\section{Acknowledgement}

The author is grateful to Angelina Hue Yi Sze, whose help with data collection was invaluable; and to Michael Apter for reading and commenting on earlier drafts of this paper. Invaluable help was given by Apter International with processing of the AMSP-R data.

\section{References}

Apter International. (2004). Research Manual for the Apter Motivational Style Profile (AMSP). Loughborough: Apter International Ltd.

Apter, M. (1989) Reversal Theory: Motivation, Emotion and Personality. London: Routledge.

Apter, M. (2015). Exploring the concept of focus in reversal theory. Journal of Motivation, Emotion and Personality, 4, 1-8.

Apter, M. \& Desselles, M. (2001). Reversal Theory Measures. In M. Apter ed. Motivational styles in everyday life: A guide to reversal theory. Washington, DC: American Psychological Association.

Apter, M., Mallows, R., \& Williams, S. (1998). The development of the motivational style profile. Personality and Individual Differences, 24, 7-18.

Bem, S. (1974). The measurement of psychological androgyny. Journal of Consulting and Clinical Psychology, 42, 155-162.

Chang, W. (1996). A research note: Gender traits in Singapore. Journal of Women and Gender Studies, 8, 221-237.

Chang, W. (1997). Values, achievement motivation and gender traits in Singapore: Implications for development. $\mathrm{Pa}$ per presented the International Conference on Women in the Asia-Pacific Region: Persons, Powers and Politics. Singapore.

Cheung, S., Cheung, F., Howard, R. \& Lim, Y.-H. (2006). Personality across the ethnic divide in Singapore: Are "Chinese Traits" uniquely Chinese? Personality and Individual Differences, 41, 467-477.

Gilmore, D. D. (1990). Manhood in the Making. Cultural Aspects of Masculinity. New York: Yale University Press.
Hamburger, M.E., Lilienfeld, S.O. \& Hogben, M. (1996). Psychopathy, gender, and gender roles: implications for antisocial and histrionic personality disorders. Journal of Personality Disorders, 10, 41-55.

Howard, R., Tay, SY, Lee HL \& Toh SM (2002). Risk taking and metamotivational state. Personality $\mathcal{E}$ Individual Differences, 32, 155-165.

Hwang, A., Francesco, A. \& Kessler, E. (2003). The relationship between individualism-collectivism, face, and feedback and learning processes in Hong Kong, Singapore, and the United States. Journal of Cross-Cultural Psychology, 34, 72-90.

Karniol, R., Gabay, R., Ochion, Y., \& Harari, Y. (1998). Is gender or gender-role orientation a better predictor of empathy in adolescence? Sex Roles, 39, 45-59.

Larsen, R.J. \& Buss, D.M. (2002). Personality Psychology: Domains of Knowledge About Human Nature. New York: McGraw Hill.

Milovchevich, D., Howells, K., Drew, N., \& Day, A. (2001). Sex and gender role differences in anger: An Australian community study. Personality and Individual Differences, 31, 117-127.

Money, J. (1992). Gendermaps: the concept of gender identity in childhood and adolescence after 37 years. Presented at a two-day multidisciplinary international conference on Gender Identity and Development in Childhood and Adolescence, St. George's Hospital, London, 13th14th March, 1992.

Severiens, S., \& Ten Dam, G. (1997). Gender and gender identity differences in learning styles. Educational Psychology, 17, 79-93.

Singapore Department of Statistics (2001). Census of population 2000: Geographic distribution and travel. Singapore: Department of Statistics.

Spence, J., \& Helmreich, R. (1983). Achievement-related motives and behaviors. In: J. Spence, ed. Achievement and achievement motives: Psychological and sociological approaches (pp. 7-74). San Francisco: Freeman.

Tabachnick, B. \& Fidell, L. (2001). Using multivariate statistics (4th ed.). Boston, MA: Allyn and Bacon.

Ward, C. (2000). Models and measurements of psychological androgyny: A cross-cultural extension of theory and research. Sex Roles, 43, 529-552.

Wharton, M., Day, A., Mohr,P., Gerace, A. \& and Howells, K. (2014). The impact of masculinity on anger arousal in ambiguous situations. Journal of Relationships Research, 5, e1. doi:10.1017/jrr.2014.1. 


\section{Appendix}

\section{Description of AMSP-R Scales}

A. Telic subscale. The sum of the scores on the five telic items. This indicates the absolute frequency of the telic state.

B. Paratelic subscale. The sum of the scores on the five paratelic items. This indicates the absolute frequency of the paratelic state.

C. Conformist subscale. The sum of the scores on the five conformist items. This indicates the absolute frequency of the conformist state.

D. Negativistic subscale. The sum of the scores on the five negativistic items. This indicates the absolute frequency of the negativistic state.

E. Self-oriented mastery. The sum of the scores on the five items that combine self-oriented and mastery.

F. Self-oriented sympathy. The sum of the scores on the five items that combine autic and sympathy.

G. Other-oriented mastery. The sum of the scores on the five items that combine other-oriented and mastery.

H. Other-oriented sympathy. The sum of the scores on the five items that combine other-oriented and sympathy.

I. Mastery subscale. The sum of the scores on the ten items that have a mastery component, divided by two to make commensurate with the other subscale scores. This indicates the absolute frequency of the mastery state.

J. Sympathy subscale. The sum of the scores on the ten items that have a sympathy component, divided by two to make commensurate with the other subscale scores. This indicates the absolute frequency of the sympathy state.

$K$. Self-oriented subscale. The sum of the scores on the ten items that have a self-oriented component, divided by two to make it commensurate with the other subscale scores. This indicates the absolute frequency of the self-oriented state.
L. Other-oriented subscale. The sum of the scores on the ten items that have an other-oriented component, divided by two to make commensurate with the other subscale scores. This indicates the absolute frequency of the other-oriented state.

M. Telic dominance. The relative importance of the telic and paratelic states, estimated by the relative frequency of the telic versus the paratelic state. This is measured by subtracting the paratelic from the telic subscale score.

N. Conformist dominance. The relative importance of the conformist and negativistic states, estimated by the relative frequency of the conformist versus the negativistic state. This is measured by subtracting the negativistic from the conformist subscale score.

O. Mastery dominance. The relative importance of the mastery and sympathy states, estimated by the relative frequency of the mastery versus the sympathy state. This is measured by subtracting the sympathy from the mastery subscale score.

P. Self-oriented dominance. The relative importance of the self-oriented and other-oriented states, estimated by the relative frequency of the self-oriented versus the otheroriented state. This is measured by subtracting the otheroriented from the self-oriented subscale score.

Q. Telic/paratelic salience. The importance of the telic/paratelic dimension taken as a whole, measured by adding the telic and the paratelic subscale scores.

R. Conformist/negativistic salience. The importance of the conformist/negativistic dimension taken as a whole, measured by adding the conformist and the negativistic subscale scores.

S. Transactional salience. The importance of the transactional states taken together, measured by adding mastery/sympathy and self-oriented/other-oriented salience. 